\title{
Perturbations in the Earth's Rotation and Geomagnetic Core-Mantle Coupling
}

\author{
M.G. ROCHESTER \\ Department of Physics, Memorial University of Newfoundland \\ St. John's, Newfoundland, Canada
}

(Received August 26, 1968)

\begin{abstract}
The effect of electromagnetic core-mantle coupling on small changes in the length of day and the geographical location of the pole is treated by a simplified model which neglects the time required for magnetic diffusion through the lower mantle, and approximates the poloidal field by the centred dipole. The resulting formulae for the time constants of the coupling are more meaningful physically than, and give nearly the same numerical values as, those derived previously by more rigorous methods. It is shown that, despite the complexity of the electromagnetic interactions at the coremantle boundary, the two kinds of perturbation in the Earth's rotation can be treated independently. Simple expressions are given for the electromagnetic restoring torque on the accelerating mantle. The effect of the geomagnetic coupling on the diurnal wobble is discussed, and concluded to be negligible.
\end{abstract}

\section{Introduction}

The detailed operation of the magnetohydrodynamic dynamo in the Earth's fluid core is still far from being understood. But by now most geophysicists are convinced that the complex pattern of internal motions in the core, together with its high electrical conductivity, permit a satisfactory explanation of the continued existence and secular variation of the geomagnetic field. Since the electrical conductivity of the lower mantle must be much less than that of the core, correspondingly weak electrical currents will be induced in it as the changing magnetic field diffuses through it toward the Earth's surface. By Lenz's law the interactions between the field and these currents will produce a variable torque coupling the mantle to the motion of the core. Fortunately it has been possible to make considerable progress in studying this electromagnetic coupling independently of any assumptions about the intricate magnetohydrodynamics of the core.

The passive response of the coupled mantle to the secular variation has been discussed by Bullard et al. (1950), Rochester (1960, hereafter referred to as I), Kakuta (1961, 1965), Rikitake (1962), Roden (1963), and Rochester \& Smylie (1965, hereafter referred to as II).*

* Runcorn (1968) remarks that "the theory of this electromagnetic coupling has not yet been fully worked out" without at all acknowledging the existence of this substantial body of published work aswell as relevant papers by Takeuchi \& Elsasser (1954) and Elsasser \& Takeuchi (1955). Indeed his only reference is to his own early introductory discussion (Runcorn 1954) of the agency of electromagnetic torques in transferring angular momentum between the core and mantle. 
In all but the last of these papers the authors were concerned with only the component of the geomagnetic coupling about the Earth's axis of figure and its relevance to the rapidity of the irregular fluctuations in the length of the day. In II we examined in detail the equatorial components of the coupling and found them to be ineffective in either exciting or damping the Chandler wobble.

All of these studies treated changes in the axial rate of rotation and in the geographical position of the pole as taking place independently of one another. However it is clear from the expressions for the components of the electromagnetic torque on the mantle (Rochester 1962) that changes in field strength which accompany the production of one component will almost necessarily give rise to the others. In this paper I give a unified treatment of small fluctuations in the three components of angular velocity of the mantle geomagnetically coupled to the core, and arrive in $\S 6$ at a numerical estimate of the extent to which polar motion and changes in the length of day are indirectly connected via the electromagnetic coupling across the core-mantle boundary.

The results of earlier work on the time constants of the coupling are confirmed in $\S 5$, by a method (outlined in $\S 3$ ) which makes the physical mechanism of damping relative accelerations between the core and mantle much more transparent than in the rigorous treatment in I and II. The limitations of this method when high-frequency excitations are involved are mentioned in $\S 7$, in connection with a discussion of the effect of electromagnetic coupling on the diurnal mode of free nutation.

The model adopted for the Earth's interior is essentially that described in II, $\S 2, \S 9$. The first few sections of the present paper review and modify the pertinent dynamical and electromagnetic theory.

\section{Core-mantle dynamics}

For dynamical purposes the rotation of the Earth is referred to geographic axes (Munk \& MacDonald 1960, pp. 11-12). This frame of reference rotates in space with an angular velocity whose components along its own axes are

$$
\Omega\left(m_{1}, m_{2}, 1+m_{3}\right)
$$

where $\Omega=7.29 \times 10^{-5} \mathrm{rad} \mathrm{sec}^{-1}$ is the mean angular speed of rotation of the Earth's crust. For brevity the complex equatorial component of any vector such as $\bar{m}$ is written

$$
\widetilde{m}=m_{1}+i m_{2}
$$

and an asterisk denotes complex conjugation. The mantle and crust are regarded as an elastic shell with equatorial and axial moments of inertia $\mathrm{A}$ and $\mathrm{C}$ respectively. In all but gyroscopic terms in the equations of motion it is sufficiently accurate to take

$$
A \simeq C \simeq 7.2 \times 10^{37} \mathrm{~kg} \mathrm{~m}^{2} .
$$

Runcorn (1954) gave the first magnetohydrodynamic argument for treating the entire core as rigid during the rapid exchange of angular momentum with the mantle. Rikitake (1962) found that an attempt to take into account the detailed magnetohydrodynamics of 
the outer core gave results for the axial coupling not significantly different from those obtained using a rigid core model. Representation of the outer core as rigid for electromagnetic calculations $(\S 3)$ simply relegates to the core-mantle boundary itself the production of toroidal field induced by relative motion between the mantle and the outer core. In line with the purpose of this paper, to use as simple a model as possible consistent with what appear to be the essential aspects of the phenomena in question, I shall retain the rigid sphere model for the core.

So far as calculation of the time constants of the coupling is concerned it is immaterial whether one:

(a) explicitly recognizes, as in I and II, the existence of the westward drift of the core past the mantle at the present average rate as an equilibrium situation (however temporary) upon which the secular variation effects perturbations; or

(b) incorporates the westward drift into the variable relative rotation between the core and mantle. In this paper I take the latter, simpler point of view, which is equivalent to treating the westward drift as a transient phenomenon as has been urged by Runcorn $(1959,1967)$. Then in conformity with the notation of II the core is regarded as a rigid sphere with moment of inertia

$$
D=\alpha A \quad(\alpha \simeq \cdot 12)
$$

and its angular velocity in space has components

$$
\Omega\left(n_{1}, n_{2}, 1+n_{3}\right)
$$

referred to the geographic axes.

There is in II an extensive discussion of core-mantle dynamics which must be generalized to allow for excitations other than purely electromagnetic. This is especially necessary because of the major result of II, that electromagnetic excitation of wobble is negligible. Therefore the mantle is prescribed to have acting on it a "modified excitation function" (Munk \& MacDonald, pp. 41-42) with "components" $\Psi_{i}$ such that

$$
\begin{aligned}
\widetilde{\Psi} & =\frac{i}{A \Omega \omega}(\widetilde{L}+\widetilde{G}+\widetilde{\Gamma})-\frac{\beta}{\omega} \dot{\tilde{m}} \\
\dot{\Psi}_{3} & =\left(L_{3}+G_{3}+\Gamma_{3}\right) / C \Omega
\end{aligned}
$$

Here $\omega=\Omega / 437$ is the circular frequency of the Chandler wobble, $\beta$ is a positive real constant associated with the non-electromagnetic mechanism in the mantle which damps the wobble (cf. II, $\S 10$ ) and $\bar{L}$ is a quantity having the dimensions of torque which embraces all nonelectromagnetic excitations (e.g. tidal. loading, redistribution of mass by earthquakes, etc.) $\bar{G}$ is the direct perturbing torque on the mantle arising from the secular variation, and $\Gamma$ denotes only that part of the electromagnetic torque on the mantle which is due to Lenz's law, i.e. the passive response torque caused by the generation of toroidal field as the mantle accelerates past the core. This is the only change of notation from II, where both $\bar{G}$ and $\bar{\Gamma}$ were combined in the one symbol $\Gamma$ (except in $\S 11$ where they were given the symbols ${ }_{(1)} \bar{\Gamma}$ and ${ }_{(2)} \bar{\Gamma}$ respectively). The excitation function on the core is restricted to the reaction 
torque due to its electromagnetic coupling to the mantle. This is consistent even with the possibility of considering bodily tidal excitation of the mantle as long as the core is regarded as a rigid sphere. Then equations (30)-(31) of II are replaced by the set

$$
\begin{gathered}
\dot{\bar{m}}-i \omega \widetilde{m}=(\widetilde{L}+\widetilde{G}+\widetilde{\Gamma}) / A \Omega+i \dot{\bar{m}} \\
\dot{m}_{3}=\left(L_{3}+G_{3}+\Gamma_{3}\right) / C \Omega \\
\dot{\tilde{n}}-i \Omega(\widetilde{m}-\tilde{n})=-(\widetilde{G}+\widetilde{\Gamma}) / D \Omega \\
\dot{n}_{3}=-\left(G_{3}+\Gamma_{3}\right) / D \Omega
\end{gathered}
$$

As was pointed out in II, $\S 9$, it is sufficiently accurate to calculate the components $\Gamma_{i}$ (which strictly speaking are referred to geographic axes more or less fixed to the mantle) in a reference frame attached to the rigid core which coincides with the geographic frame only at the instant $t=0$.

\section{Toroidal field production at the core-mantle boundary}

For electromagnetic calculations the crust and mantle are taken to be a rigid spherical shell (radii $b=3470 \mathrm{~km}, d=6370 \mathrm{~km}$ ) slipping past a rigid outer core. The shell is an insulator above radius $c=5470 \mathrm{~km}$, and has uniform electrical conductivity $\sigma_{m}=10^{2} \mathrm{ohm}^{-1} \mathrm{~m}^{-1}$ below this radius. Roden's work suggests that the tightness of the axial coupling can be increased by a factor of five by allowing the thickness of the conducting part of the mantle to decrease while its (uniform) conductivity increases so as to maintain the same screening. However this concentration of the mantle's conductivity in a highly conducting thin layer next to the core seems an implausible extreme, so I stick to the distribution used in I and II. The conductivity of the outer core greatly exceeds $\sigma_{m}$.

The dynamical response of the mantle to a perturbation in its state of rotation is in principle a complicated one because the spatio-temporal configuration of the magnetic field coupling it to the core is not simple. Nevertheless, as the studies cited in $\S 1$ have shown, it is possible to describe the time dependence of the response by a small number of time constants each of which is characteristic of a particular mode of acceleration. Bullard et al. gave (without derivation) a formula for the relaxation time of the axial coupling which could have been obtained only by neglecting the time required for magnetic diffusion through the mantle, i.e. by assuming that changes in field strength reaching the core-mantle boundary from below are instantaneously propagated to the Earth's surface rather than retarded by the eddy currents these changes induce in the lower mantle. The nature of this approximation was pointed out in I and the tightness of the axial coupling was found to be increased by at least $20 \%$ when diffusion was taken into account. As Rikitake (1966, p. 101) mentions, neglect of diffusion is only a fair approximation because the relaxation time of the axial coupling is less than an order of magnitude greater than the time required for diffusion of a change in field strength through the mantle (Runcorn 1955, Smylie 1965). However this approximation saves tedious analysis and extensive computation, and for these reasons was used by Roden in estimating the effect of assuming a somewhat more realistic model for the distribution of electrical conductivity with depth in the mantle than had been 
used by other workers. For the sake of simplifying the mathematics as much as possible I neglect magnetic diffusion in this paper.

The velocity of the mantle past the core, referred to axes fixed to the outer core, is

$$
\sum_{k=-1}^{1} \Delta q_{1}{ }^{k} e^{i k \phi}\left(\frac{i k}{\sin \theta} \hat{\theta}-\hat{\phi} \frac{d}{d \theta}\right) P_{1}{ }^{k}(\cos \theta) .
$$

Here the standard orientation of unit vectors in spherical polar coordinates, and the associated Legendre function defined by Cołson (1935), are used. As was shown in II, $\S 9$,

$$
\begin{aligned}
& \Delta q_{1}{ }^{-1}=-2 \Delta q_{1}{ }^{1 *}=b \Omega(\tilde{m}-\tilde{n}) \\
& \Delta q_{1}{ }^{0}=b \Omega\left(m_{3}-n_{2}\right)
\end{aligned}
$$

to the same order of accuracy as (1)-(2), i.e. to the first order in the small quantities $m_{i}, n_{i}$.

The effect of neglecting magnetic diffusion is that in order to calculate the toroidal fields induced at the core-mantle boundary by the relative motion there, these fields may be taken as stationary with respect to the core and established instantaneously by that motion. The disadvantage of using the geographic frame for electromagnetic calculations is that fields stationary with respect to the outer core are time dependent in the frame of reference: (Smylie 1965). If, as in II, axes attached to the outer core are used, then $\partial / \partial t=0$ for fields stationary with respect to the core but there are induction terms in the equation governingthe establishment of these fields in the mantle, due to the sweep of the mantle past these fields. For the purposes of this paper equation (6b) of II can be written

$$
\begin{gathered}
{\left[\frac{1}{r} \frac{d^{2}}{d r^{2}}(r)-\frac{n(n+1)}{r^{2}}\right] t_{n}{ }^{m}=\mu_{0} \sigma_{m}\left[\frac{i}{2 b}(n-m)(n+m+1) \Delta q_{1}{ }^{-1} t_{n}{ }^{m+1}\right.} \\
\left.+\frac{i m}{b} \Delta q_{1}{ }^{0} t_{n}{ }^{m}-\frac{i}{b} \Delta q_{1}{ }^{1} t_{n}{ }^{m-1}\right]
\end{gathered}
$$

where $t_{n}{ }^{m}(r)$ is a harmonic coefficient of the toroidal field induced at the core-mantle boundary, defined in equations (4)-(5) of II. The necessary boundary conditions (from equations (8)-(9) of II) are

$$
t_{n}^{m}(c)=0
$$

and

$$
\begin{aligned}
{\left[\frac{d}{d r}\left(r t_{n}{ }^{m}\right)\right]_{r=b^{+}} } & =-\mu_{0} \sigma_{m}\left[\Delta q _ { 1 } { } ^ { - 1 } \left\{\frac{(n-1)(n-m-1)(n-m)}{2(2 n-1)} s_{n-1}{ }^{m+1}\right.\right. \\
& \left.+\frac{(n+2)(n+m+1)(n+m+2)}{2(2 n+3)} s_{n+1}{ }^{m+1}\right\} \\
& +\Delta q_{1}{ }^{0}\left\{\frac{(n-1)(n-m)}{2 n-1} s_{n-1}{ }^{m}-\frac{(n+2)(n+m+1)}{2 n+3} s_{n+1}{ }^{m}\right\} \\
& \left.+\Delta q_{1}{ }^{1}\left\{\frac{n-1}{2 n-1} s_{n-1}{ }^{m-1}+\frac{n+2}{2 n+3} s_{n+1}{ }^{m-1}\right\}\right]
\end{aligned}
$$

where the poloidal harmonic coefficients $s_{n}{ }^{n}$ are evaluated at $r=b$.

The obvious difficulty in finding a solution to (4) when the relative rotation between core and mantle is about an axis inclined to the axis of figure is that the toroidal coeffi- 
cients become entangled, with $t_{n}{ }^{m}$ depending on $t_{n}{ }^{m \pm 1}$. One way of getting around the difficulty would be to transform to a new set of spherical polar coordinates with polar axis parallel to the instantaneous axis of relative rotation, and proceed as in II, $\S 5$. But then one has to work out the transformation of the resulting toroidal harmonic coefficients back to the original coordinate system, using e.g. the formulae given by Jeffreys (1965). This seems a formidable task, and I have instead devised an approximate solution for the situation where the (steady) relative rotation is at a rate comparable with the observed wobble and westward drift. This is given in the Appendix. The result is that, to within an error of at most $10 \%,(4)-(5)$ are satisfied by

$$
t_{n}^{m}(b) \simeq-f_{n}\left[\frac{d}{d r}\left(r t_{n}{ }^{m}\right)\right]_{r=b^{+}}
$$

where

$$
f_{n}=\left[1-\left(\frac{b}{c}\right)^{2 n+1}\right] /\left[n+(n+1)\left(\frac{b}{c}\right)^{2 n+1}\right]
$$

To the same degree of accuracy the poloidal harmonic coefficients at $r=b$ can be written in terms of the standard Schmidt coefficients (II, § 5):

$$
\begin{aligned}
s_{n}{ }^{m} & \simeq-\frac{d}{2 n}\left(\frac{d}{b}\right)^{n+1}(-1)^{1 / 2(m+|m|)} . \\
& \cdot\left[\left(2-\delta_{m 0}\right) \frac{(n-m) !}{(n+m) !}\right]^{1 / 2}\left[g_{n}^{|m|}\left(1+\delta_{m 0}\right) \mp i h_{n}^{|m|}\right]
\end{aligned}
$$

according as $m$ is positive or negative.

It was shown in I that taking into account the poloidal harmonics up to the sixth increases the tightness of the axial coupling by $40 \%$ from the value obtained by approximating the poloidal part of the geomagnetic field in the mantle by the centred dipole alone. However the dipole approximation so simplifies both the mathematical expressions and their physical interpretation that I adopt it in this paper. Then the only toroidal fields induced at $r=b$ are those with coefficients $t_{2}{ }^{m}$, and (6)-(7) reduce to

$$
t_{2}{ }^{m}(b) \simeq \frac{\mu_{0} \sigma_{m} f_{2}}{3}\left[\frac{(1-m)(2-m)}{2} \Delta q_{1}{ }^{-1} s_{1}{ }^{m+1}+(2-m) \Delta q_{1}{ }^{0} s_{1}{ }^{m}+\Delta q_{1}{ }^{1} s_{1}{ }^{m-1}\right]
$$

and (9) gives

$$
\begin{gathered}
s_{1}{ }^{-1}=-2 s_{1}{ }^{1 *} \simeq-\left(g_{1}{ }^{1}+i h_{1}{ }^{1}\right) \frac{d^{3}}{b^{2}} \\
s_{1}{ }^{0} \simeq-g_{1}{ }^{0} \frac{d^{3}}{b^{2}}
\end{gathered}
$$

where the Schmidt coefficients have the values

$$
\left.\begin{array}{l}
g_{1}{ }^{0} \\
g_{1}{ }^{1} \\
h_{1}{ }^{1}
\end{array}\right\}=\left\{\begin{array}{l}
-3.055 \\
-0.227 \\
+0.590
\end{array}\right\} \times 10^{-5} \text { weber } \mathrm{m}^{-2}
$$




\section{Electromagnetic restoring torque on the mantle}

In conformity with the neglect of magnetic diffusion the instantaneous Lenz-law torque on the mantle must be calculated as if the mantle moves past the core uniformly at the instantaneous rate, and about the instantaneous axis, of relative rotation. From II, $\S 4$

$$
\begin{aligned}
\widetilde{\Gamma}= & -\frac{4 \pi b^{2}}{\mu_{0}} \sum_{n \geqslant 1} \sum_{m=-n}^{n}(-1)^{m} \frac{n(n+1)}{2 n+1} s_{n}{ }^{m}\left[\frac{n+2}{2 n+3} t_{n+1}{ }^{-m-1}+\frac{n-1}{2 n-1} t_{n-1}{ }^{-m-1}\right] \\
\Gamma_{3}= & -\frac{4 \pi b^{2}}{\mu_{0}} \sum_{n \leqslant 1} \sum_{m=-n}^{n}(-1)^{m} \frac{n(n+1)}{2 n+1} s_{n}{ }^{m}\left[\frac{(n+2)(n-m+1)}{2 n+3} t_{n+1}{ }^{-m}\right. \\
& \left.-\frac{(n-1)(n+m)}{2 n-1} t_{n-1}{ }^{-m}\right]
\end{aligned}
$$

where the poloidal and toroidal harmonic coefficients are evaluated at $r=b$, and given therefore by (10)-(11). In the dipole approximation to the poloidal field these expressions for torque reduce, with the aid of (3), to

$$
\begin{aligned}
& \widetilde{\Gamma} \simeq-D \Omega\left[\gamma(\widetilde{m}-\tilde{n})-(\chi+i \Psi)(\widetilde{m}-\tilde{n})^{*}+(\zeta-i \eta)\left(m_{3}-n_{3}\right)\right] \\
& \Gamma_{3} \simeq-D \Omega\left[\frac{1}{2}(\zeta+i \eta)(\widetilde{m}-\tilde{n})+\frac{1}{2}(\zeta-i \eta)(\widetilde{m}-\tilde{n})^{*}+\frac{\kappa}{1+\alpha}\left(m_{3}-n_{3}\right)\right]
\end{aligned}
$$

where for convenience the following symbols have been defined:

$$
\begin{aligned}
& \left.\begin{array}{l}
r \\
\chi \\
\Psi \\
\zeta \\
\eta \\
\kappa
\end{array}\right\}=\frac{4 \pi \sigma_{m} f_{2} d^{6}}{15 D b}\left\{\begin{array}{l}
6\left(g_{1}{ }^{0}\right)^{2}+7\left[\left(g_{1}{ }^{1}\right)^{2}+\left(h_{1}{ }^{1}\right)^{2}\right] \\
\left(h_{1}{ }^{1}\right)^{2}-\left(g_{1}{ }^{2}\right)^{2} \\
-2 g_{1}{ }^{1} h_{1}{ }^{1} \\
2 g_{1}{ }^{0} g_{1}{ }^{1} \\
-2 g_{1}{ }^{0} h_{1}{ }^{1} \\
(1+\alpha)\left\{8\left(g_{1}{ }^{0}\right)^{2}+6\left[\left(g_{1}{ }^{1}\right)^{2}+\left(h_{1}{ }^{1}\right)^{2}\right]\right\}
\end{array}\right. \\
& =\left\{\begin{array}{l}
4.4 \\
0.02 \\
0.02 \\
0.10 \\
0.27 \\
6.4
\end{array}\right\} \times 10^{-10} \mathrm{sec}^{-1}
\end{aligned}
$$

upon substituting the numerical values for the various symbols.

\section{Relaxation times for changes in the rotation of the mantle}

Let the Laplace transform operator be $\int_{0}^{\infty} e^{-p t} d t$ and define $\mu_{i}, \nu_{i}, l_{i}, g_{i}$ as the transforms of $m_{i}, n_{i}, L_{i}, G_{i}$ respectively. The transform of $\widetilde{m}^{*}$ can be written $\tilde{\mu}^{*}$ as long as it is understood that the complex variable $p$ is not affected by complex conjugation (cf. II, § 9). Upon substituting (12) and taking the Laplace transform, the set (1)-(2) can be written

$$
[p(1-i \beta)-i \omega] \tilde{\mu}+\alpha\left[\gamma(\tilde{\mu}-\tilde{\nu})-(\chi+i \Psi)(\tilde{\mu}-\tilde{\nu})^{*}+(\zeta-i n)\left(\mu_{3}-\nu_{3}\right)=\frac{\tilde{l}+\tilde{g}}{A \Omega}\right.
$$




$$
\begin{aligned}
& p \mu_{3}+\alpha\left[\frac{1}{2}(\zeta+i n)(\tilde{\mu}-\tilde{\nu})+\frac{1}{2}(\zeta-i n)(\tilde{\mu}-\tilde{\nu})^{*}+\frac{\kappa}{1+\alpha}\left(\mu_{3}-\nu_{3}\right)\right]=\frac{l_{3}+g_{3}}{C \Omega} \\
& -p \tilde{\mu}+(p+i \Omega+\gamma)(\tilde{\mu}-\tilde{\nu})-(\chi+i \Psi)(\tilde{\mu}-\tilde{\nu})^{*}+(\zeta-i n)\left(\mu_{3}-\nu_{3}\right)=\frac{\tilde{g}}{\alpha A \Omega} \\
& -p \mu_{3}+\frac{1}{2}(\zeta+i n)(\tilde{\mu}-\nu)+\frac{1}{2}(\zeta-i n)(\tilde{\mu}-\tilde{\nu})^{*}+\left(p+\frac{\kappa}{1+\alpha}\right)\left(\mu_{3}-\nu_{3}\right)=\frac{g_{3}}{\alpha C \Omega}
\end{aligned}
$$

together with the complex conjugates of (14a) and (15a).

After a great deal of algebra, details of which I omit to save space, the set (14)-(15) can be solved to obtain explicit representations of each of the six variables $\left.\tilde{\mu}, \tilde{\mu}^{*}, \mu_{3}, \tilde{\mu}-\tilde{\nu}\right)$, $(\tilde{\mu}-\tilde{\nu})^{*}, \mu_{3}-\nu_{3}$ as linear functions of the excitations. A considerable reduction in the amount of algebra required is effected by recognizing that the determinant of the matrix of the coefficients in (14)-(15), given the symbol $A$, can be easily factored by knowing where the poles of $\mu_{i}$ are situated in the complex p-plane. These poles will be grouped in pairs, located as follows. A real pair, associated with changes in the length of day, will be at $p=0$ and $p=-\frac{1}{\tau_{3}}$, where $\tau_{3}$ is the relaxation time of the axial coupling. One complex pair will be at $p \simeq \pm i \omega-\frac{1}{\tau}$, where $\tau$ is the damping time of wobble at the Chandler frequency. As is well known from the work of Poincaré (1910) and others (cf. §7) the mantle should theoretically ke able to exhibit free nutation of nearly diurnal frequency because of its inertial coupling to the ellipsoidal liquid core. In the model of core-mantle interaction considered in this paper electromagnetic coupling of the mantle to a rigid spherical core has the same effect, i.e. another complex pair of poles should be found near $p= \pm i \Omega$.

It turns out that the determinant $\Delta$ is given by

$$
\left(1+\beta^{2}\right)^{-1} \Delta=\left(p-p_{1}\right)\left(p-p_{1}^{*}\right)\left(p-p_{2}\right)\left(p-p_{2}^{*}\right) p\left(p+\frac{1}{\tau_{3}}\right)
$$

where, to the first order in the small quantities $\left(\frac{\omega}{\Omega}\right)^{2}, \frac{\gamma}{\Omega}, \ldots, \frac{\kappa}{\Omega}, \beta^{3}$ :

$$
\begin{gathered}
p_{1} \simeq i \omega\left(1-\beta^{2}\right)-\frac{1}{\tau} \\
p_{2} \simeq-i \Omega(1+s)-\frac{1}{\tau_{2}} \\
\frac{1}{\tau} \simeq \beta \omega\left(1-\beta^{2}\right)+\frac{\alpha \omega \gamma}{\Omega} \\
\frac{1}{\tau_{3}} \simeq \kappa \\
s \simeq \frac{\alpha \beta \gamma}{\Omega} \\
\frac{1}{\tau_{2}} \simeq \gamma\left[1+\alpha\left(1-\frac{\omega}{\Omega}-\beta^{2}\right)\right]
\end{gathered}
$$

Let $\tau \simeq 15 \mathrm{yr}$ in (18), then $\beta \simeq 0.013$, which justifies neglecting terms of order higher than $\beta^{3}$. The core, through electromagnetic coupling to the mantle, contributes only a fraction 


$$
\frac{\alpha \omega r \tau}{\Omega} \simeq 6 \times 10^{-5}
$$

of the damping of the Chandler wobble. This confirms a result of II.

When the great contrast in electrical conductivity between the core and mantle is taken into account, the expression for the relaxation time of the axial coupling given by Bullard et al. is equivalent to (19), except that the coupling due to the equatorial components of the poloidal dipole is included here. Using (13), (19) gives

$$
\tau_{3} \simeq 50 \mathrm{yr}
$$

only a factor of two greater than the result of a rigorous analysis (I).

\section{Cross-coupling between changes in the rotation of the mantle}

It is obvious from the form of (14)-(15) that in principle the three components of change in the rotation of the mantle cannot be treated independently of one another, and that any mechanism which generates one component will indirectly give rise to others, through the passive electromagnetic coupling. As the mantle responds to excitation of one component $m_{i}$, toroidal field is produced by slip at the core-mantle boundary. The electrical currents in the mantle supporting this toroidal field, and the field itself, interact with the existing poloidal field and its associated current system to provide a Lorentz torque on the mantle. In general this torque will not be entirely about the axis of the component $m_{i}$ originally excited, so other components are generated. Thus it is legitimate to speak of, e.g., axial torques on the mantle producing wobble! The term "cross-coupling" can be used to describe this connection.

The importance of this cross-coupling can be estimated from the solutions of (14)-(15) referred to in $\S 5$. Retaining only terms of first order in the small quantities $\frac{\gamma}{\Omega}$, etc.

$$
\begin{aligned}
& A \Omega(1-i \beta)\left(p-p_{1}\right) \tilde{\mu} \simeq\left(p-p_{2}\right)^{-1}[(p+i \Omega+\gamma) \tilde{l}+(p+i \Omega) \tilde{g}] \\
& \quad+\alpha(\chi+i \Psi)\left(p-p_{2}{ }^{*}\right)^{-1}\left[\tilde{l}+\left(\frac{1+\alpha}{\alpha}\right) \tilde{g}\right]^{*} \\
& \quad-\alpha(\zeta-i n)\left(p+\frac{1}{\tau_{3}}\right)^{-1}\left[l_{3}+\left(\frac{1+\alpha}{\alpha}\right) g_{3}\right]
\end{aligned}
$$

The contribution of the cross-coupled excitations to wobble at the Chandler frequency can be compared to that of the direct excitation by setting $p \simeq i \omega$ in the factors on the RHS of (22) and substituting numerical values from (13). Even the largest cross-coupled excitation is only $10^{-4}$ as effective as the direct excitation in generating the Chandler wobble.

There is no need to give here the full solution for $\mu_{3}$ corresponding to (22). Equatorial excitations are at most $10^{-6}$ as competent as axial ones to generate changes in the length of day, so the cross-coupling is even weaker than in the case of wobble.

Taking higher harmonics of the poloidal field into account will not significantly change these results. It is therefore possible to conclude that the cross-coupling between changes in the rotation of the mantle is negligible, and that the excitation of polar motion and of 
changes in the axial rate of rotation can be treated independently, as in I and II.

This conclusion simplifies the mathematics considerably, because it permits setting $\chi$, $\Psi, \zeta, \eta$ equal to zero. Thus the passive response torque (12) can be written

$$
\begin{aligned}
& \widetilde{\Gamma} \simeq-\gamma D \Omega(\widetilde{m}-\tilde{n}) \\
& \Gamma_{3} \simeq-\frac{\kappa}{1+\alpha} D \Omega\left(m_{3}-n_{3}\right)
\end{aligned}
$$

It is worth noting that the characteristic frequencies and time constants of the coupled core-mantle system, given by (16)-(21), are independent of $\chi, \Psi, \zeta, \eta$ to the first order, and so are not affected by the approximation (23). In this approximation (22) reduces to

$$
A \Omega(1-i \beta) \tilde{\mu} \simeq \frac{(p+i \Omega+\gamma) \widetilde{l}+(p+i \Omega) \tilde{g}}{\left(p-p_{1}\right)\left(p-p_{2}\right)}
$$

and the remaining members of (14)-(15) yield

$$
\begin{aligned}
C \Omega_{\mu_{3}} & \simeq \frac{\left(p+\frac{\kappa}{1+\alpha}\right) l_{3}+p g_{3}}{p\left(p+\frac{1}{\tau_{3}}\right)} \\
A \Omega(1-i \beta)(\tilde{\mu}-\tilde{\nu}) & \simeq \frac{\tilde{p} \widetilde{l}+[p(1+\alpha-i \beta)-i \omega] \frac{\tilde{g}}{\alpha}}{\left(p-p_{1}\right)\left(p-p_{2}\right)} \\
C \Omega\left(\mu_{3}-\nu_{3}\right) & \simeq \frac{l_{3}+\left(\frac{1+\alpha}{\alpha}\right) g_{3}}{p+\frac{1}{\tau_{3}}}
\end{aligned}
$$

The set (24)-(25) constitute a satisfactory basis for discussing the effect of any low-frequency excitation which perturbs the Earth's rotation.

\section{Diurnal wobble}

In the Chandler motion the Earth wobbles as a whole, but the existence of the liquid core provides an additional degree of freedom so that the core and mantle can be regarded as coupled oscillators constituting a dynamical system with two normal modes, of which the Chandler wobble is only one. The second mode, with nearly diurnal period, was first predicted by Hough and Sluodsky independently in 1895. The theory of this phenomenon has been extended by Poincaré (1910), Jeffreys \& Vicente (1957) and Molodenskii (1961). In the diurnal mode, in contrast to the Chandler wobble, there is substantial relative motion between the core and mantle.

In all these studies the core-mantle coupling is effected by inertial forces arising from the distribution of pressure exerted by the rotating liquid core on its ellipsoidal boundary with the mantle, and electromagnetic coupling is entirely ignored. The difference between the period of diurnal wobble and the sidereal day is of the order of the ellipticity of the core-mantle boundary, i.e. about 200-300 sec.

In this paper, on the other hand, the core-mantle boundary is regarded as spherical, thus 
eliminating the possibility of inertial coupling, and the coupling mechanism is entirely electromagnetic. As was seen in $\S 5$ the diurnal mode predicted for purely electromagnetic coupling has a period differing from the sidereal day by only

$$
\frac{2 \pi s}{\Omega} \simeq 10^{-3} \mathrm{sec} .
$$

The effect of electromagnetic coupling on the period of the diurnal free nutation is negligible compared with that of the core's ellipticity. The work of $\S 5$ indicates that the diurnal wobble is very weakly damped by the electromagnetic coupling, whereas no dissipation is provided by inertial coupling to an inviscid liquid core. The predicted damping time, given by (21),

$$
\tau_{2} \simeq \frac{1}{\gamma(1+\alpha)} \simeq 65 \mathrm{yr}
$$

is about $10^{4}$ periods.

Popov (1963) claims to have detected the diurnal mode in observations of latitude variation made at Poltava over two decades. The amplitude is of the order of $0^{\prime \prime} .01$, about a tenth that of the Chandler wobble. This is so small as to be almost undetectable, and the amplitude calculated may be critically affected by any error in the theoretical value of the period (Thomas 1964). Pariiskii (1963) has pointed out that the period predicted by Molodenskii's theory is close to resonance with the $K_{1}$ wave of the lunisolar diurnal tide.

It is interesting to estimate the amplitude of the diurnal free nutation which this resonance could excite, using (24a). Since

$$
\frac{p+i \Omega+r}{\left(p-p_{1}\right)\left(p-p_{2}\right)} \simeq \frac{1+\frac{i \alpha \gamma}{\Omega}}{p-p_{1}}-\frac{\frac{i \alpha \gamma}{\Omega}}{p-p_{2}}
$$

the diurnal part of the wobble is found by inverting

$$
\begin{array}{r}
A \Omega \tilde{\mu} \simeq-\frac{i \alpha \gamma}{\Omega} \frac{\tilde{l}}{p-p_{2}} \\
\therefore \quad \widetilde{m} \simeq-\frac{i \alpha \gamma}{A \Omega^{2}} \int_{0}^{t} \widetilde{L}(\lambda) e^{p_{2}(t-\lambda)} d \lambda
\end{array}
$$

The factor $\frac{\alpha r}{\Omega}$ explains why the amplitude of diurnal wobble due to low-frequency excitations such as the secular variation is negligible and could be ignored in II. For excitation by the lunisolar tidal torque take

$$
\begin{aligned}
\widetilde{L} \simeq-\frac{3 G}{4} & (C-A)\left(\frac{M_{\odot}}{R_{\odot}{ }^{3}}+\frac{M_{\mathbb{C}}}{R_{\mathbb{C}}^{3}}\right) \sin 2 \Theta e^{-i \Omega t} \\
& \simeq-(1+\alpha) A \Omega P \sin \Theta e^{-i \Omega t}
\end{aligned}
$$

where $P=7.74 \times 10^{-12} \mathrm{rad} \mathrm{sec}^{-1}$ is the rate of steady precession of the Earth's axis in space, and $\Theta=23 \frac{1}{2}^{\circ}$ is the obliquity of the ecliptic. The free wobble so excited has amplitude

$$
\frac{\alpha \gamma(1+\alpha) P \sin \Theta}{\Omega}\left|i \Omega s+\frac{1}{\tau_{2}}\right|^{-1} \simeq \frac{\alpha P \sin \Theta}{\Omega} \simeq 0^{\prime \prime} .001,
$$


an order of magnitude less than Popov's ralue.

The accuracy of this estimate is impaired by the fact that inertial coupling may be much more important than electromagnetic, for motions tending to produce relative wobble between the core and mantle (cf. Toomre 1966, who gives a related discussion of the comparative importance of these two coupling mechanisms in precession). Also, as Pariiskii suggests, the large relative wobble in the diurnal mode implies a reduction in the effectiveness of the electromagnetic coupling at such high frequencies. This is to be expected because the time required for toroidal field produced at the core-mantle boundary to significantly penetrate the lower mantle and couple it to the core must be of the order of a year (Smylie 1965). The neglect of retardation of magnetic diffusion is probably a serious shortcoming in the above analysis of the effect of electromagnetic coupling on the diurnal nutation. Progress is being made in an attempt to extend Poincaré's treatment of coremantle dynamics by taking into consideration electromagnetic, as well as inertial, coupling and will be reported in a later paper.

\section{Relative motion between the core and mantle}

The motion of the core past the mantle, as viewed from the geographic frame of reference, is described by inverting (25). Without going into details, a few comments can be made.

First, the direct exciting torque due to the secular variation is $\left(\frac{1+\alpha}{\alpha}\right)$ times as effective in generating relative motion between the core and mantle as in changing the state of rotation of the mantle itself. Physically this factor is to be expected because the core's rotational inertia is so much less than the mantle's that the exchange of angular momentum across their boundary affects the angular velocity of the former much more than the latter.

Comparing (25b) with (24b) it is clear that the axial component of relative motion has the same relaxation time as a change in the length of day. This was first noted by Bullard et al. It was shown in I that the electromagnetic coupling alone is sufficient to damp the axial acceleration of the mantle. On the other hand it has been shown (first in II, and confirmed in $\S 5$ of the present paper) that electromagnetic damping is inadequate by a factor of at least $10^{4}$ to dissipate the energy of the Chandler wobble. Because $\frac{\omega}{\Omega}, \beta \ll 1$ it is sufficiently accurate to write (25a) as

$$
A \Omega(\tilde{\mu}-\tilde{\nu}) \simeq \frac{\omega}{\Omega}\left(\frac{\tilde{l}+\tilde{g}}{p-p_{1}}\right)+\frac{\tilde{l}+\left(\frac{1+\alpha}{\alpha}\right) g}{p-p_{2}}
$$

As expected ( $\$ 7$ ), the relative wobble is dominated by the diurnal mode and its damping is due entirely to the electromagnetic coupling. It is interesting that $\tau_{2}$, given by (26), is comparable with the axial time constant $\tau_{3}$. Taking into account the higher poloidal harmonics and allowing for magnetic diffusion should not reduce $\tau_{2}$ by as much as an order of magnitude. 


\section{Gyroscopic effects on excitation of the Chandler wobble}

The effectiveness of the electromagnetic coupling in damping the Chandler wobble is lower than that with which it brakes relative wobble between the core and mantle by a factor

$$
\frac{\alpha \omega \gamma \tau_{2}}{\Omega} \simeq\left(\frac{\alpha}{1+\alpha}\right) \frac{\omega}{\Omega}
$$

This is clearly a gyroscopic effect due to the Earth's equatorial bulge. Such factors are of course crucial to a proper discussion of polar motion and their neglect leads to grave errors in the treatment of the subject, as noted e.g. by Elsasser \& Munk (1958) and by Munk \& Mac Donald (p. 57).

A recent example of work nullified by exactly such a mistake is Runcorn's (1968) attempt to refute the demonstration by Mansinha \& Smylie (1967) that earthquakes probably can generate the Chandler wobble. Runcorn proceeds further to offer a superficial argument in support of electromagnetic excitation of the wobble, which not only fails to take into account the vital gyroscopic factor but also omits any reference whatever to the opposite conclusion arrived at earlier in a series of independent studies (Elsasser \& Munk, Munk \& Hassan (1961), II) all of which did include the gyroscopic effect.

\section{Conclusions}

The main conclusions from the work presented in this paper can be summarized as follows :

(i) the indirect "cross-coupling" between changes in the axial and equatorial components of the Earth's rotation is sufficiently weak that these changes can be treated independently;

(ii) the degree of confirmation of previous, more rigorous, calculations of the time constants of the electromagnetic coupling implies that neglect of the retardation of the diffusion of changes in magnetic field through the accelerating mantle is not a bad approximation, at least for low-frequency excitations;

(iii) in this approximation the components of the electromagnetic restoring torque on the mantle can be taken as proportional to the corresponding components of the angular velocity of the mantle relative to the core;

(iv) electromagnetic coupling damps the diurnal free nutation only very weakly and is negligible compared with inertial coupling in affecting the period.

Though it is to be expected (cf. § 7) that electromagnetic core-mantle coupling will not greatly influence the short-period wobble and bodily tides, the possibility of a detectable effect needs to be more carefully investigated. The effect on precession is still unresolved, and is the subject of current research mentioned at the end of $\S 7$. 


\section{Acknowledgements}

I am grateful to Dr. D.E. Smylie for many helpful discussions, and to Dr. F.D. Stacey for raising stimulating questions, during the course of preparation of this paper.

\section{Appendix}

Toroidal field generated by steady slip between core and mantle

Define the operator

$$
D_{n}=\frac{1}{r} \frac{d^{2}}{d r^{2}}(r)-\frac{n(n+1)}{r^{2}}
$$

and the symbols

$$
\begin{gathered}
H_{n}{ }^{m}=-i \mu_{0} \sigma_{m}(n-m)(n+m+1) \Delta q_{1}{ }^{-1} / 2 b \\
I^{m}=-i m \mu_{0} \sigma_{m} \Delta q_{1}{ }^{0} / b \\
J=i \mu_{0} \sigma_{m} \Delta q_{1}{ }^{1} / b
\end{gathered}
$$

Then (4) becomes

$$
D_{n} t_{n}{ }^{m}=-\left[H_{n}{ }^{m} t_{n}{ }^{m+1}+I^{m} t_{n}{ }^{m}+J t_{n}{ }^{m-1}\right]
$$

Form the linear combination $\sum_{m=-n}^{n} a^{m} t_{n}{ }^{m}(r)$, operate on it with $D_{n}$, and seek the eigenvalues $-k^{2}$. A set of $2 n+1$ homogeneous linear equations for the $2 n+1$ unknowns $a^{m}$ results:

$$
-a^{m-1} H_{n}{ }^{m}+\left(k^{2}-I^{m}\right) a^{m}-J a^{m+1}=0
$$

A non-trivial solution is obtained only if the determinant of the coefficients vanishes. This gives an equation of degree $2 n+1$ in $k^{2}$ which will in general have $2 n+1$ distinct complex roots $k_{j}{ }^{2}$. To each root corresponds a set $a_{j}{ }^{m}(m=-n, \ldots, n)$ determined to within some constant factor by (A1).

$$
\therefore \quad\left(D_{n}+k_{j}^{2}\right) \sum_{m=-n}^{n} a_{j}{ }^{m} t_{n}{ }^{m}(r)=0
$$

and $\sum_{m=-n}^{n} a_{j}^{m} t_{n}{ }^{m}(r)$ is a linear combination of spherical Bessel functions. The boundary conditions (5)-(6) require that

$$
\sum_{m=-n}^{n} a_{j}{ }^{m} t_{n}{ }^{m}(r)=\frac{N_{n}{ }^{j}\left(k_{j} r\right)}{r\left[\frac{d N_{n}^{j}\left(k_{j} r\right)}{d r}\right]_{r=b}} \sum_{l=-n}^{n} a_{j}{ }^{l}\left[\frac{d}{d r}\left(r t_{n}{ }^{l}\right)\right]_{r=b^{+}}
$$

where the function $N_{n}{ }^{j}\left(k_{j} r\right)$ is defined in II, $\S 5$. These $2 n+1$ linear equations in the toroidal harmonic coefficients can be solved:

$$
t_{n}{ }^{m}(r)=\frac{1}{r} \sum_{l=-n}^{n}\left[\frac{d}{d r}\left(r t_{n}{ }^{l}\right)\right]_{r=b^{+}} \sum_{l=1}^{2 n+1} e_{j}{ }^{m} a_{j}{ }^{l} \frac{N_{n}{ }^{j}\left(k_{j} r\right)}{\left[\frac{d N_{n}{ }^{j}\left(k_{j} r\right)}{d r}\right]_{r=b}}
$$

There is no need to obtain the explicit (and complicated) expressions for the coefficients $e_{j}{ }^{m}$. By differentiating (A2),

$$
\left[\frac{d}{d r}\left(r t_{n}^{m}\right)\right]_{r=b^{+}}=\sum_{l=-n}^{n}\left[\frac{d}{d r}\left(r t_{n}^{l}\right)\right]_{r=b^{+}} \sum_{j=1}^{2 n+1} e_{j}{ }^{m} a_{j}{ }^{l}
$$




$$
\therefore \quad \sum_{j=1}^{2 n+1} e_{j}^{m} a_{j}^{l}=\delta^{m l}
$$

This removes the indeterminacy of the $a_{j}{ }^{l}$.

The quantity of interest is $t_{n}{ }^{m}(b)$. The series expansions for the Bessel functions permit writing

$$
N_{n}^{j}\left(k_{j} b\right)=-f_{n} b\left[\frac{d N_{n}^{j}\left(k_{j} r\right)}{d r}\right]_{r=b}\left\{1+0\left(k_{j} b\right)^{2}\right\}
$$

The algebraic details need not be displayed, buit the omitted terms are of the order $\left|k_{j} b\right|^{2} / 2 n^{2}$. Since $\left|k_{j} b\right|^{2}$ is the modulus of a root of a secular equation in which the determinant has elements whose moduli are all $\leqslant \frac{1}{2} n(n+1) \mu_{0} \sigma_{m} b^{2} \Omega\left|m_{i}-n_{i}\right|_{\max } \simeq 0 \cdot 1 n^{2}$ it seems likely that $\left|k_{j} b\right|^{2} \leq 0 \cdot 2 n^{2}$, i.e. that to within an error of at most $10 \%$,

$$
N_{n}{ }^{j}\left(k_{j} b\right) \simeq-f_{n} b\left[\frac{d N_{n}{ }^{j}\left(k_{j} r\right)}{d r}\right]_{r=b}
$$

independent of $j$. Hence to this accuracy, using (A3),

$$
t_{n}{ }^{m}(b) \simeq-f_{n} \sum_{l=-n}^{n}\left[\frac{d}{d r}\left(r t_{n}^{l}\right)\right]_{r=b^{+}} \sum_{j=1}^{2 n+1} e_{j}^{m} a_{j}^{l} \simeq-f_{n}\left[\frac{d}{d r}\left(r t_{n}{ }^{m}\right)\right]_{r=b^{+}}
$$

This expression is exact when $m=0$ and $\Delta q_{1}^{ \pm 1}=0$ (cf. II, $\S 5$ ) and its accuracy has been checked numerically in a couple of more general cases.

\section{References}

Bullard, E.C., C. Freedman, H. Gellman \& J. Nixon, The westward drift of the Earth's magnetic field, Phil. Trans. Roy. Soc. London, A, 243, 67-91, 1950.

Copson, E.T., Theory of Functions of a Complex Variable (Oxford University Press), 1935.

Elsasser, W.M. \& W.H. Munk, Geomagnetic drift and the rotation of the Earth, in H. Benioff et al. (eds.) Contributions in Geophysics: In Honour of Beno Gutenberg (Pergamon Press, Oxford), 228-236, 1958.

Elsasser, W.M. \& H. Takeuchi, Nonuniform rotation of the Earth and geomagnetic drift, Trans. Amer. Geophys. Union, 36, 584-590, 1955.

Jeffreys, B.S., Transformation of tesseral harmonics under rotation, Geophys. J., 10, 141-145, 1965.

Jeffreys, H. \& R.O. Vicente, The theory of nutation and the variation of latitude, M.N. Roy. Astron. Soc., 117, 142-173, 1957.

Kakuta, C., The magnetic torque on the impulsive change of the rotation of the Earth, Publ. Astron. Soc. Japan, 13, 361-368, 1961.

Kakuta, C., Note on the relaxation time of magnetic coupling in the Earth's rotation, Publ. Astron. Soc. Japan, 17, 337-338, 1965.

Mansinha, L. \& D.E. Smylie, Effect of earthquakes on the Chandler wobble and the secular pole shift, J. Geophys. Res., 72, 4731-4743, 1967.

Molodenskii, M.S., The theory of nutation and diurnal Earth tides, Comm. Obs. Roy. Belgique, No. 188, 25-56, 1961.

Munk, W.H. \& E.S.M. Hassan, Atmospheric excitation of the Earth's wobble, Geophys. J., 4, 339-358, 1961.

Munk, W.H. \& G.J.F. MacDonald, The Rotation of the Earth (Cambridge University Press), 1960. 
Pariiskii, N.N., The discovery of the Earth's diurnal nutation, Sov. Astron., 7, 424-426, 1963.

Poincaré, H., Sur la précession des corps déformables, Bull. Astron., Paris, 27, 321-356, 1910.

Popov, N.A, Nutational motion of the Earth's axis, Nature, 198, 1153-1155, 1963.

Rikitake, T., Poleward increase of the secular drift of geomagnetic field, Geomagnetica (Servico Meteorologico Nacional, Lisbon), 5-13, 1962.

Rikitake, T., Electromagnetism and the Earth's Interior (Elsevier, Amsterdam), 1966.

Rochester, M.G., Geomagnetic westward drift and irregularities in the Earth's rotation, Phil. Trans. Roy. Soc. London, A, 252, 531-555, 1960 (referred to as I in the text).

Rochester, M.G., Geomagnetic core-mantle coupling, J. Geophys. Res., 67, 4833-4836, 1962.

Rochester, M.G. \& D.E. Smylie, Geomagnetic core-mantle coupling and the Chandler wobble, Geophys. $J ., 10,289-315,1965$ (referred to as II in the text).

Roden, R.B., Electromagnetic core-mantle coupling, Geophys. J., 7, 361-374, 1963.

Runcorn, S.K., The Earth's core, Trans. Amer. Geophys. Union, 35, 49-63, 1954.

Runcorn, S.K., The electrical conductivity of the Earth's mantle, Trans. Amer. Geophys. Union, 36, 191198, 1955.

Runcorn, S.K., On the theory of the geomagnetic secular variation, Ann. de Géophys., 15, 87-92, 1959.

Runcorn, S.K., On the rotation of Jupiter, in W. Hindmarsh et al. (eds.) Magnetism and the Cosmos (Oliver \& Boyd, Edinburgh), 365-377, 1967.

Runcorn, S.K., Polar wandering and continental drift, in W. Markowitz \& B. Guinot (eds.) Continental Drift, Secular Motion of the Pole, and Rotation of the Earth (D. Reidel, Dordrecht), 80-85, 1968.

Smylie, D.E., Magnetic diffusion in a spherically-symmetric conducting mantle, Geophys. J., 9, 169-184, 1965.

Takeuchi, H. \& W.M. Elsasser, Fluid motions near the core boundary and the irregular variations in the Earth's rotation, J. Phys. Earth, Tokyo, 2, 39-44, 1954.

Thomas, D.V., Evidence for a nearly diurnal term in the nutation of the Earth's axis, Nature, 201, 481, 1964.

Toomre, A., On the coupling of the Earth's core and mantle during the 26,000 year precession, in B.G. Marsden \& A.G.W. Cameron (eds.) The Earth-Moon System (Plenum Press, New York), 33-45, 1966. 